\title{
The mass ratio distribution of B-type visual binaries in the Sco OB2 association ${ }^{\star, \star \star}$
}

\author{
N. Shatsky ${ }^{1,2}$ and A. Tokovinin ${ }^{3,1}$ \\ 1 Sternberg Astronomical Institute, Universitetskii pr. 13, Moscow 119899, Russia \\ 2 Royal Observatory of Belgium, Av. Circulaire 3, Bruxelles 1180, Belgium \\ 3 Cerro-Tololo Inter-American Observatory, La Serena, Casilla 603, Chille \\ e-mail: atokovinin@ctio.noao.edu
}

Received 25 September 2001 / Accepted 30 October 2001

\begin{abstract}
A sample of 115 B-type stars in the Sco OB2 association is examined for existence of visual companions in the $J$ and $K_{\mathrm{s}}$ bands, using the ADONIS near-infrared adaptive optics system and coronograph. Practically all the components in the separation range $0^{\prime \prime} 3-6^{\prime \prime} 4$ (45-900 AU) and magnitudes down to $K=16$ were detected. The $K$ and $J-K$ photometry of the primaries and differential photometry and astrometry of the 96 secondaries are presented. Ten secondaries are new physical components, as inferred from the photometric and statistical criteria, while the rest of the newly detected objects are faint background stars. After a small correction for detection incompleteness and a conversion of the fluxes into masses, an unbiased distribution of the components mass ratio $q$ was derived. The power law $f(q) \propto q^{-0.5}$ fits the observations well, whereas a $q^{-1.8}$ distribution, which corresponds to a random pairing of stars, is rejected. The companion star fraction is $0.20 \pm 0.04$ per decade of separation which is comparable to the highest measured binary fraction among low-mass PMS stars and $~ 1.6$ times higher than the binary fraction of low-mass dwarfs in the solar neighborhood and in open clusters in the same separation range.
\end{abstract}

Key words. binaries: visual - stars: statistics - formation

\section{Introduction}

Binary star formation mechanisms represent an important but still poorly understood part of star formation. A concerted effort is therefore necessary to fill this gap from both theoretical and observational sides.

Observationally, multiplicity statistics in stellar populations of different environment, age and mass are one of the most important clues to binary formation. Considerable data have been accumulated for old lowmass solar-type nearby stars (Duquennoy \& Mayor 1991 hereafter DM91), for pre-main sequence (PMS) stars and for the stars of intermediate mass and age in open clusters (as reviewed by Duchêne 1999). In contrast, the multiplic-

Send offprint requests to: N. Shatsky,

e-mail: kolja@sai.msu.ru

* Based on observations collected at the European Southern Observatory, La Silla, Chile (ESO programme 65.H-0179).

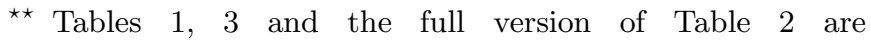
only available in electronic form at the CDS via anonymous ftp to cdsarc.u-strasbg.fr $(130.79 .128 .5)$ or via http://cdsweb.u-strasbg.fr/cgi-bin/qcat?J/A+A/382/92 ity properties of high-mass stars remain poorly known. The most recent comprehensive study of B-type stars still seems to be that of Abt et al. (1990), based on spectroscopic data and traditional catalogues of visual binaries. The effort of Brown \& Verschueren (1997) to measure precise radial velocities of B-type stars in Sco OB2 has not yet provided updated statistics of short-period systems. Here we study the binarity and mass ratio distribution of B-type stars in the separation range of 45-900 AU.

The discovery of massive visual binaries is limited to a mass ratio $q$ close to 1 because of the high intrinsic brightness of B-type primaries. Recently, a speckleinterferometric survey of O-type stars was done by Mason et al. (1998). However, despite the increased angular resolution, only a few new pairs were discovered, owing to the magnitude-difference restrictions of optical interferometry. On the other hand, interferometry in the infra-red (IR) has led to the discovery of 4 additional companions to the 4 brightest stars in the Orion Trapezium (Weigelt et al. 1999). Söderhjelm $(1997,2001)$ obtained an unbiased distribution of the mass ratio of $\mathrm{A}$ and $\mathrm{F}$ binary stars from the magnitude differences measured by Hipparcos, 


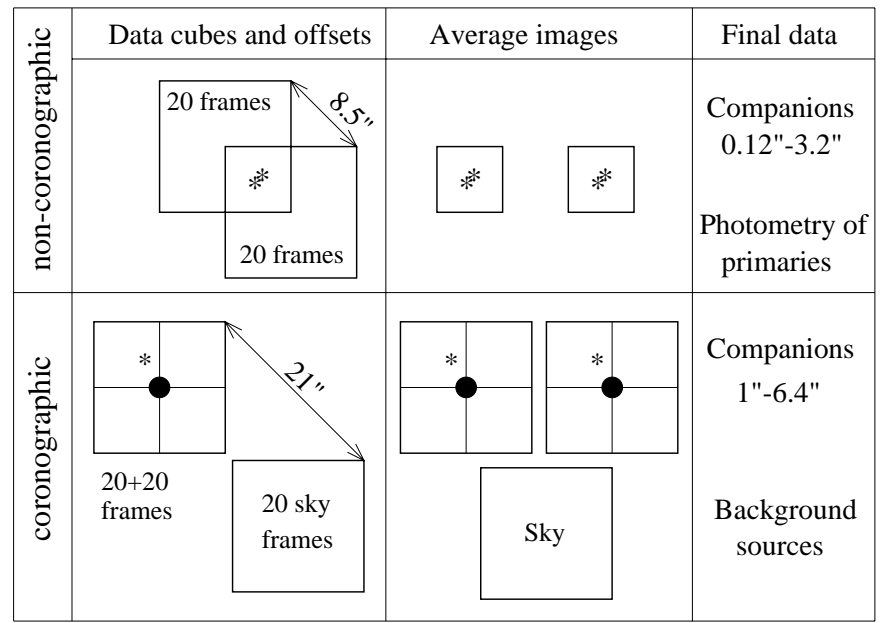

Telescope

Instrument

Camera, detector

Image format

Coronographic mask

Filters $(\lambda / \Delta \lambda, \mu \mathrm{m})$ :

One-frame exposure

ESO 3.6 m, La Silla, Chile

ADONIS AO system

Sharp II + NICMOS3

$256^{2} 0^{\prime \prime} .05$ pixels, or $\left(12^{\prime \prime} .8\right)^{2}$

Radius $1^{\prime \prime}$

$J(1.25 / 0.3), K_{\mathrm{s}}(2.15 / 0.3)$

0.05-0.4 s (non-coronographic)

$3-5$ s (coronographic)

Fig. 1. Scheme of ADONIS (Beuzit et al. 1997) data and basic instrumental parameters.

but only for $q>0.6$, where detection was complete. For A-type stars, $f(q)$ is roughly uniform in this interval at short (60-120 AU) separations and slightly rises towards small $q$ at larger (240-480 AU) separations.

In 1997 we used the ESO $3.6 \mathrm{~m}$ telescope with coronograph to investigate the advantages that Adaptive Optics (AO) offers for the detection and study of low-mass companions to B-type stars (Tokovinin et al. 1999 - TCSB99). The companion detection was complete in the separation range from $1^{\prime \prime}$ to $6^{\prime \prime}$ and for magnitude differences up to $10^{\mathrm{m}}$ and more in the $K$ photometric band. The high dynamic range imaging and the reduced luminosity ratio in the $K$ band (compared to the visible) give access to companions with masses down to the bottom of the Main Sequence and below, thus permitting for the first time a complete companion census and an unbiased mass ratio distribution in the accessible range of separations. The use of AO helps to reduce the residual wings of the Point Spread Function (PSF) outside the coronographic mask, but, more importantly, concentrates the light from secondary companions into the diffraction-limited image cores, thus greatly improving their detectability against the primary component's wings. Bouvier et al. (1997, 2001) have already used AO to study binarity in open clusters.

Low-mass binary companions to B-type stars are sometimes detected by their X-ray emission. This method is complementary to AO imaging: it is sensitive to all separations but can not detect components with the lowest masses (see discussion in TCSB99). Recently,
Hubrig et al. (2001) observed a sample of X-ray selected stars of late B spectral type with $\mathrm{AO}$ and detected new optical and physical components. They did not derive improved binary statistics from these data.

In this paper we probe for binarity a homogeneous sample of 115 B-type stars in the Sco OB2 association. This association is ideally suited for the studies of B-stars binary properties for several reasons. It is among the closest to the Sun $(d=145 \mathrm{pc})$, has a well-defined age with small spread (from 4 to $15 \mathrm{Myr}$ for the different sub-groups, de Zeeuw et al. 1999), and is relatively well investigated in many respects (de Geus et al. 1989). Practically all B-type stars were observed by Hipparcos which provided secure membership status and additional constraints on their binarity from the astrometry. The binary statistics of low-mass PMS stars in Sco OB2 is available for comparison (Köhler et al. 2000; Brandner et al. 1996). Thus, it is possible to check the theoretical predictions about the dependence of binary statistics on primary mass.

In Sect. 2 we describe the observing method and the characteristics of our sample of B-type stars. In Sect. 3 we outline the data processing, derive the limits of companion detection and provide the $J$ and $K$ photometry of the primaries and their known and newly discovered companions. The interpretation of our results follows in Sect. 4, where we derive the mass ratio distribution. Finally, the results are discussed and compared to other works in Sect. 5.

\section{Statistical sample and observations}

Our list of targets is based on the work of Brown \& Verschueren (1997) who provided recent data for OB-type stars in the Sco OB2 association. The membership of these stars in association is confirmed by the Hipparcos data (de Zeeuw et al. 1999). A few additional targets were also selected from the latter work.

Eight visual binaries with separations from $1^{\prime \prime}$ to $6^{\prime \prime}$ and small magnitude difference were removed from the observational program because they were not suitable for wave-front sensing. These objects were included in the final statistical analysis, however. We presumed that there are no additional companions to these stars in the studied separation range, because most of such companions would be dynamically unstable (TCSB99). The basic data for the target stars are given in Table 1 . The interstellar extinction, which is generally small, was either taken from de Geus et al. (1989) or estimated from the $(B-V)$ color. Note that some pairs of targets belong to the same wide multiple systems; nevertheless, they were observed and analyzed independently, as described below.

The observations were performed from $24 / 25$ to 28/29 May, 2000. For each target star, we obtained a sequence of images (the so called data cubes) in the $J$ and $K_{\mathrm{s}}$ (hereafter $K$ ) filters. Data with and without 
coronographic mask were taken in each filter (Fig. 1):

1. Short integration time images ( $T_{\text {int }}$ limited by saturation of the detector) were taken without the coronograph in " $1 / 4$ frame" mode: the image of the target star was placed in the center of one of the detector quadrants for the first half of the data cube acquisition, and then, with the help of the chopping mirror of ADONIS, shifted to the opposite quadrant for the rest of cube. Binaries with separations of $0{ }^{\prime \prime} 12-33^{\prime \prime} 2$ can be observed in this mode, with partial coverage up to $9^{\prime \prime}$.

2. Long integration time cubes were taken with the target star placed in the center of the detector field of view and hidden by a coronographic mask of $1^{\prime \prime}$ radius. In this mode, a sequence of sky frames was taken between two sequences of object frames. The covered range of separations is thus $1^{\prime \prime}-6$ ". 4 . With the coronograph, the detection limit at moderate separations is about $2^{\mathrm{m}}-3^{\mathrm{m}}$ deeper than in direct images.

The seeing and transparency during this run were variable; the periods of photometric conditions covered only partially the first and the second nights and the whole last night. During the last night, most of the targets with newly found companions were re-observed to secure their photometric parameters. For target stars observed only in non-photometric conditions, the $K$ and $J-K$ values in Table 1 are flagged accordingly.

\section{Data processing and results}

In this section we describe the processing of the data cubes, the search for and measurement of companions, and the photometry of both secondary and primary components.

\subsection{Primary data reduction}

For the primary data reduction, we used the Eclipse package (Devillard 1997), since it includes the special utility for the processing of images in the ADONIS format. The standard initial steps of the data processing included subtraction of average sky frames (for the noncoronographic mode - subtraction of the frames with a target star in the opposite quadrant), division of the result by the flat field and correction for bad pixels. Flat fields were taken on the dusk sky as in TCSB99. The photometric precision after flat field division is $1-2 \%$.

Two series of 20 individual images in each data cube (both coronographic and direct) were averaged to produce two independent final images per cube (Fig. 1), or a total of 8 average images for each target in two filters and in two modes. The subsequent reduction was done independently to assure the reality of the detected components and to assess the precision of photometric and position parameters. While reducing the data cubes, additional information of two kinds was also obtained:

Sky offset fields. To estimate the surface density of field stars around each target, we have processed separately

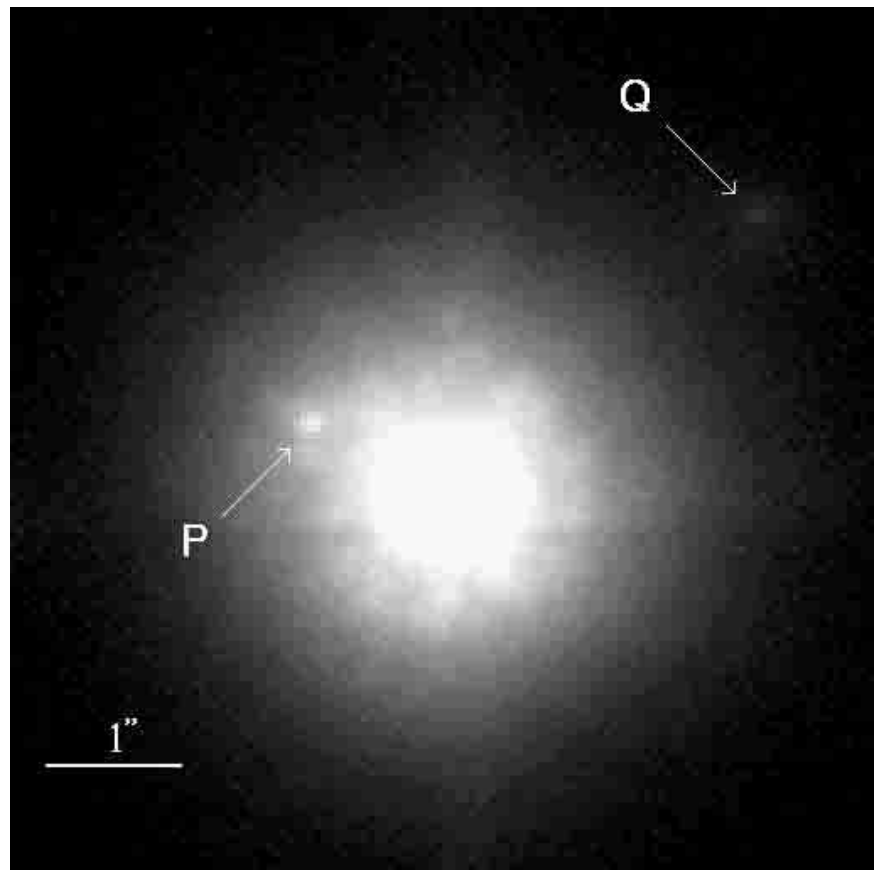

Fig. 2. The non-coronographic image of HD 144987 with two new companions $\mathrm{P}$ and $\mathrm{Q}$. The wings of the PSF have the speckle structure. The primary component is saturated in the given intensity scale.

the sky offset images obtained in coronographic mode. The subtraction of the dark current and background was done with the help of cleaned background images. Latter frames were obtained by median averaging of the sky offset frames of several targets taken with the same filter and integration time, thus eliminating all stars. No flat field division was performed on the sky images.

Plane-by-plane flux variations. As one of the diagnostics of non-photometric conditions, we computed the variation of the integral flux from the target star between different planes of the non-coronographic data cubes. Any variances above 5\% were treated as due to clouds.

\subsection{Detection of companions}

The search for faint point sources in the vicinity of intrinsically bright B-type target stars is a non-trivial task. The Point Spread Function (PSF) of ADONIS normally consists of a sharp (diffraction-limited) core and extended wings with a characteristic speckle pattern (Fig. 2). This pattern changes from object to object and represents a major obstacle for detection of faint companions in $\mathrm{AO}$ images (e.g. Racine et al. 1999). We tried to reduce speckle noise on non-coronographic images by subtracting PSF models as described in Appendix A and achieved noise levels some 1.5-2 times lower. Speckle structure in the ADONIS images is not random (as assumed in Racine et al.) but indeed semi-static. 


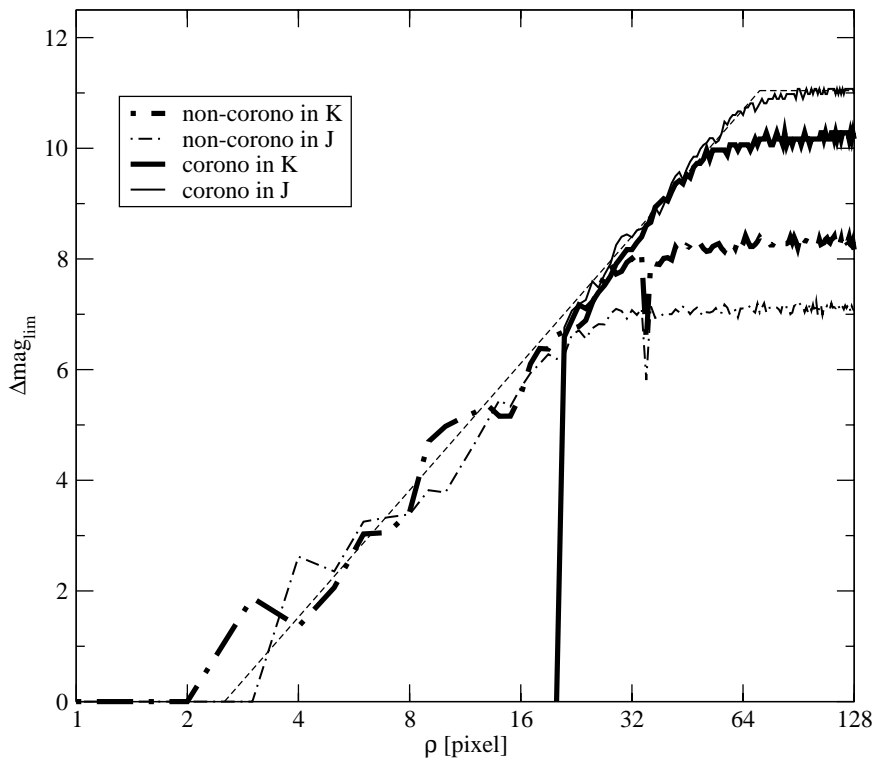

Fig. 3. The detection curves for the non-coronographic and coronographic images of HD 100546 in the $J$ and $K$ bands. Coronographic curves start at $\rho=20$ pixels. The log-linear fit with a saturation plateau is used to approximate the combined curve in $\mathrm{K}$ (dashed line). The "spike" at $\rho=35$ pixels is caused by subtraction of the averaged PSF fitted by DAOPHOT (Appendix A).

We developed a special code jupe (to be included in the new distribution of Eclipse) to determine the radial profile $P(\rho)$ of the PSF, considering that the target star is hidden by the coronograph mask. First, the position of the star is found by some centering of the PSF wings in the $x$ and $y$ directions (cf. TCSB99). The radial distance $\rho$ is measured from this position. The profile $P(\rho)$ is the median value of the intensity at a given $\rho$. We used the same code to remove the average radial residuals from the PSF-subtracted non-coronographic images.

Images with subtracted $P(\rho)$ were searched for point sources using the findobjs algorithm from Eclipse. The detection threshold was set to $3 \sigma(\rho)$, where $\sigma(\rho)$ is the rms of the azimuthal intensity fluctuations in the PSF wings at each $\rho$, also computed by the jupe procedure. These thresholds were converted from pixel intensities to integrated fluxes with the help of Strehl ratios measured on non-coronographic images. In this way, the limiting magnitude differences $\Delta J_{\lim }(\rho)$ and $\Delta K_{\lim }(\rho)$ were derived for each frame (see example in Fig. 3).

The detection limits for the whole sample have some common characteristics. At small $\rho$, they are approximately proportional to $R=\lg \rho$, with the average slope $\mathrm{d} K_{\lim } / \mathrm{d} R=7^{\mathrm{m}} 1 \pm 0.8$. Further on, they saturate at some level which depends on the integration time. In coronographic images $K_{\lim }$ saturates at $K=16.8 \pm 0.5$ which is about $2^{\mathrm{m}}-3^{\mathrm{m}}$ fainter than in non-coronographic images. It is possible to describe the detection limit by a merged curve which consists of the non-coronographic linear part for $\rho \leq 1^{\prime \prime}$ and coronographic part for $\rho>1^{\prime \prime}$. This curve, typically, is continuous at the junction point $\rho=1^{\prime \prime}$
(Fig. 3). In other words, in the area close to the mask edge the residual speckle noise in a coronographic image with subtracted $P(\rho)$ and in the respective non-coronographic image with subtracted average PSF (Appendix A) are roughly the same. Individual log-linear slopes and saturation levels of the merged detection curves were found for each target star. They were subsequently converted into limiting mass ratios $q_{\lim }(\rho)$ (Sect. 4.4).

\subsection{Photometry of primary components}

The majority of the targets in our sample did not have any reliable measurements of near-IR magnitudes and colors before our study. To measure the flux, one should integrate the signal in an aperture as large as possible, reducing the dependence of the result on the image quality. The efficiency of the AO correction changed significantly, as the seeing was varying from 0.55 to $2{ }^{\prime \prime} .0$. The result should also be be insensitive to detector imperfections and to the presence of other sources in the field of view. We computed the flux of the target star as

Flux $=\sum_{\rho<2^{\prime \prime}} I(x, y)+\int_{2^{\prime \prime}}^{5^{\prime \prime}} P(\rho) 2 \pi \rho \mathrm{d} \rho$,

where the first term is the sum of the pixel values $I(x, y)$ inside a circle of $2^{\prime \prime}$ radius and the second term is the integral of the median radial profile. The median averaging effectively removes all distant $\left(\rho>2^{\prime \prime}\right)$ sources and detector defects. For binary targets with $\rho<2^{\prime \prime}$ the flux of each component was then computed from the total flux and the magnitude difference as found by PSF fitting.

The integration of the median profile $P(\rho)$ gives a somewhat lower flux than the direct integration of intensity, because the average intensity of speckles is higher than their median intensity. Nevertheless, tests have shown that this bias is less than $1 \%$ (or $0.01^{\mathrm{m}}$ ).

The magnitudes were reduced to zenith with average extinction coefficients of 0.08 in $J$ and 0.10 in $K$. The errors caused by extinction uncertainty are negligible because all objects were observed close to zenith. The photometric zero points were determined from the primary standard star HD 161743 and confirmed by 5 other stars for which $J$ and $K$ magnitudes were taken from Simbad. All determinations are mutually consistent to within $\pm 5 \%$.

The resulting $K$ magnitudes and $J-K$ colors of the primary components are given in Table 1 . The errors reflect the deviations of the individual fluxes from the mean and also take into account the flux difference between two halves of the data cubes (Sect. 3.1). Observations under non-photometric conditions are marked by "c" in the flags column. For these targets, the measured $J$ and $K$ magnitudes represent only upper limits, whilst we adopted the lowest of the measured magnitudes.

We computed the expected magnitudes $K_{\text {theo }}$ based on the spectral types and visual magnitudes of all sample stars. These estimates are in good agreement with the 
actual data for the majority of targets measured in photometric conditions: the difference $K_{\text {obs }}-K_{\text {theo }}$ shows a rms scatter of 0.15 and its absolute value is less than 0.4 for all targets but two.

These two outliers with $K$ excess of about $1^{\mathrm{m}}$ are HD 100546 and HD 143275. The first star is reported to harbor a significant amount of circumstellar dust (Augereau et al. 2001; Meeus et al. 2001), a natural source of higher infrared luminosity. The second star ( $\delta$ Sco) was intensively studied and its photometry from Simbad $(K=2.75)$ agrees much better with $K_{\text {theo }}$ than our own measurement $(K=1.85)$. Our result could possibly be explained by an error of ADONIS shutter timing at short $(0.02 \mathrm{~s})$ exposure. HD 143275 is the brightest star in our sample. Nevertheless, other bright stars were also observed with such integration time and the estimated random shutter error does not exceed $0.003 \mathrm{~s}$ for them.

On the other hand, $\delta$ Sco is a multiple star with a Betype primary and complex light variations. An extended study is published by Otero et al. (2001) where the observations of a " $\gamma$ Cas-like outburst" are reported. The peak magnitude of 1.9 in the visible was detected just two months after our observations. The authors explain this event as being caused by the periastron passage in a close multiple star. This is a second, more attractive astrophysical explanation of our discrepant photometry.

Based on a comparison of observed and expected $K$ magnitudes, we have extended the validity of companion's photometry to 16 stars which were observed on nonphotometric nights. They have $\left|K_{\text {obs }}-K_{\text {theo }}\right|<0.3$ and their $J-K$ colors deviate from the estimated by less than 0.06 . These cases are marked as "+" in the flags column of Table 1. We estimate that the external errors of our photometry are not larger than $\pm 00^{\mathrm{m}} 1$ in $K$ and $\pm 0^{\mathrm{m}} 15$ in $J-K$.

\subsection{Photometry and astrometry of companions}

The program findobjs provides the approximate positions and brightness of the detected sources. The final measurement of their relative coordinates and magnitudes was made with the profile-fitting utility NSTAR of the DAOPHOT package (Stetson 1987). The image of the primary star (if single) taken without coronograph was selected as a PSF model for fitting distant $\left(\rho>1^{\prime \prime} .75=\right.$ 35 pixels) components, whereas synthetic average PSFs (Appendix A) were used for closer pairs. The positions of primaries on the coronographic images were inferred by indirect techniques with reduced accuracy (Appendix B). The characteristic error in position is $00^{\prime \prime} 005-00^{\prime \prime} 010$.

The relative component positions in pixel coordinates were transformed into arcseconds. For calibration, we used the binary stars HD 120709 and HD 199005 AB measured by Hipparcos. The pixel size was found to be $0^{\prime \prime} 0497 \pm$ 0 !' 0005 , and the orientation of the detector rows was found to be east-west to within \pm 0 : 1 . Measurements of several additional known binaries confirmed this calibration.

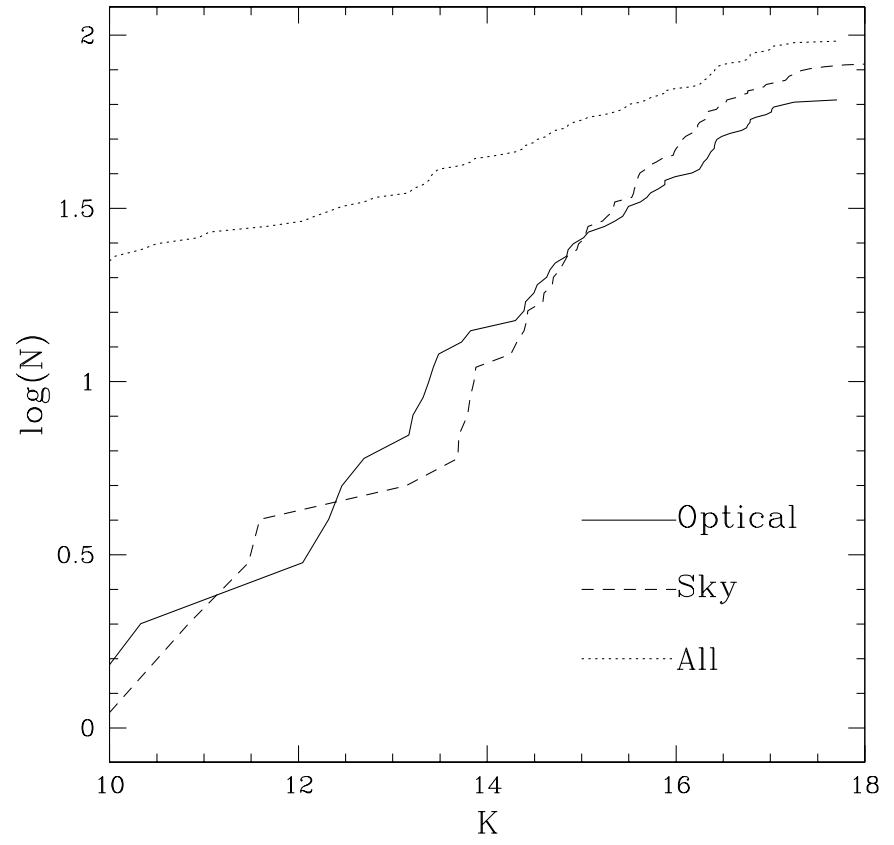

Fig. 4. Cumulative distributions of $K$ magnitudes of all the sources found around the targets (dotted line) and in the sky frames (dashed line). The distribution of sources in the target frames which are considered as optical (Sect. 4.2) is plotted in solid line and resembles the dashed curve of the sky-frame background sources.

The magnitudes and relative positions of the $96 \mathrm{sec}-$ ondary components are given in Table 2 (its full version is published electronically).

The approximate (as given by findobjs) magnitudes of some one hundred field stars found in the sky frames are provided in Table 3. These data are used to estimate the surface density of the background stellar population. In both tables the $K$ magnitudes and $J-K$ colors are given with their errors. The errors are inferred from the scatter of the individual values obtained from the measurement of different frames. The errors in Table 2 are those of magnitude differences and do not include uncertainties of the primary star magnitudes. The cumulative distributions of $K$ magnitudes of sources around targets and in the sky frames are shown in Fig. 4. The majority of the sources are faint, close to the detection limit at $K \approx 16^{\mathrm{m}}$.

Few bright binaries from Table 2 were measured by Hipparcos or Tycho (ESA, 1997) in the visible. The comparison of their magnitude differences in the $K$ band with $\Delta V_{\mathrm{T}}$ or $\Delta \mathrm{Hp}$ is shown in Fig. 5.

Four stars from our sample (HD 100841, 113902, 126981, 145483) were also observed by Hubrig et al. (2001). They did not detect the bright companion to HD 100841. The reality of this component is dubious since it is not seen in our $J$-band images either. On the other hand, an additional close ( $\left(0^{\prime \prime} 2\right)$ companion to HD 145483 was found by Hubrig et al.; we did not observe this target because it has a known companion at $33^{\prime \prime} 8$. Another suspicious component is HD $133937 \mathrm{P}$, which is not seen in $J$ but quite prominent in our sole $K$-band image. Both the 
Table 2. Photometry and differential astrometry of the companion stars. Only the data for the observed non-optical components are shown here, together with the primary $K$-magnitude taken from Table 1 . The last column gives the status of the companions (see text), new companions are marked; "?" in the Sys column denotes uncertain detections. Field flag contains "c" for data obtained in non-photometric conditions or "+" for likely photometric conditions (Sect. 3.3); ":" denotes incomplete resolution.

\begin{tabular}{|c|c|c|c|c|c|c|c|c|c|c|c|c|c|}
\hline $\mathrm{HD}$ & Sys & $K_{1}$ & $K_{2}$ & $\epsilon_{\Delta K}$ & $(J-K)_{2}$ & $\epsilon_{\Delta(J-K)}$ & flag & $\rho\left({ }^{\prime \prime}\right)$ & $\epsilon_{\rho}\left({ }^{\prime \prime}\right)$ & $\theta\left(^{\circ}\right)$ & $\epsilon_{\theta}\left({ }^{\circ}\right)$ & $N_{\text {obs }}$ & Status \\
\hline 98718 & $\overline{A B}$ & 4.66 & 5.86 & 0.01 & -0.03 & 0.01 & & 0.354 & 0.005 & 143.6 & 0.5 & 1 & $\mathrm{P}$ \\
\hline 100841 & $\mathrm{AP} ?$ & 3.17 & 6.81 & 0.27 & & & + & 0.734 & 0.038 & 135.2 & 3.0 & 1 & P new \\
\hline 104878 & $\mathrm{AB}$ & 5.67 & 7.00 & 0.02 & 0.07 & 0.08 & $\mathrm{c}$ & 0.698 & 0.008 & 157.9 & 0.2 & 2 & $\mathrm{P}$ \\
\hline 108250 & $\mathrm{AP}$ & 5.36 & 10.50 & 0.03 & 1.01 & 0.05 & + & 2.362 & 0.024 & 53.2 & 0.1 & 1 & $\mathrm{P}$ new \\
\hline 109668 & $\mathrm{AP}$ & 3.41 & 10.94 & 0.26 & 0.82 & 0.29 & $\mathrm{c}$ & 4.853 & 0.049 & 198.3 & 0.1 & 3 & P? new \\
\hline 113703 & $\mathrm{AP}$ & 5.80 & 9.16 & 0.02 & 0.47 & 0.02 & $\mathrm{c}$ & 1.551 & 0.016 & 268.2 & 0.2 & 2 & P new \\
\hline 116087 & $\mathrm{AB}$ & 5.12 & 7.03 & 0.07 & -0.11 & 0.23 & & 0.164 & 0.010 & 135.2 & 3.6 & 2 & $\mathrm{P}$ \\
\hline 120324 & $\mathrm{AP}$ & 3.97 & 10.06 & 0.05 & 1.02 & 0.18 & $\mathrm{c}$ & 4.637 & 0.047 & 304.2 & 0.1 & 2 & $\mathrm{P}$ new \\
\hline 120709 & $\mathrm{AB}$ & 5.05 & 6.32 & & 0.02 & & & 7.878 & 0.079 & 105.8 & 0.1 & 1 & $\mathrm{P}$ \\
\hline 130807 & $\mathrm{AB}$ & 5.27 & 6.84 & 0.01 & -0.12 & 0.08 & c: & 0.099 & 0.008 & 86.0 & 7.3 & 1 & $\mathrm{P}$ \\
\hline 131120 & $\mathrm{AP}$ & 5.54 & 9.43 & 0.10 & 0.85 & 0.12 & & 1.046 & 0.012 & 161.1 & 0.4 & 2 & $\mathrm{P}$ new \\
\hline 132200 & $\mathrm{AC}$ & 4.09 & 5.46 & 0.04 & -0.04 & 0.04 & & 0.128 & 0.008 & 156.4 & 1.9 & 1 & $\mathrm{P}$ \\
\hline 132200 & $\mathrm{AB}$ & 4.09 & 8.45 & 0.03 & 0.61 & 0.03 & & 3.950 & 0.040 & 83.0 & 0.1 & 2 & $\mathrm{P}$ \\
\hline 133937 & $\mathrm{AP} ?$ & 7.16 & 11.05 & 0.02 & & & $\mathrm{c}$ & 0.006 & 0.048 & 293.2 & 0.1 & 1 & $\mathrm{P}$ new \\
\hline 136504 & $\mathrm{AB}$ & 4.28 & 5.55 & 0.06 & 0.42 & 0.12 & + & 0.279 & 0.008 & 149.2 & 1.0 & 1 & $\mathrm{P}$ \\
\hline 140008 & $\mathrm{AB}$ & 5.79 & 9.47 & 0.05 & 0.42 & 0.05 & $\mathrm{c}$ & 0.507 & 0.009 & 132.8 & 0.8 & 1 & $\mathrm{P}$ \\
\hline 142378 & $\mathrm{AB}$ & 6.13 & 7.78 & 0.01 & 0.23 & 0.02 & & 0.524 & 0.006 & 119.7 & 0.5 & 1 & $\mathrm{P}$ \\
\hline 144217 & $\mathrm{AB}$ & 3.86 & 6.80 & 0.05 & -0.78 & 0.12 & $\mathrm{c}$ & 0.292 & 0.010 & 170.5 & 3.1 & 1 & $\mathrm{P}$ \\
\hline 144218 & $\mathrm{AE}$ & 5.96 & 7.43 & 0.05 & & & c: & 0.119 & 0.005 & 36.3 & 2.5 & 1 & $\mathrm{P}$ \\
\hline 144987 & $\mathrm{AP}$ & 5.74 & 9.75 & 0.08 & 0.57 & 0.12 & & 1.119 & 0.015 & 116.9 & 0.3 & 5 & P new \\
\hline 144987 & $\mathrm{AQ}$ & 5.74 & 12.81 & 0.06 & 1.04 & 0.26 & & 3.056 & 0.032 & 228.0 & 0.2 & 3 & $\mathrm{P} ?$ new \\
\hline 145502 & $\mathrm{AB}$ & 4.20 & 5.14 & 0.01 & 0.06 & 0.01 & + & 1.334 & 0.014 & 1.8 & 0.1 & 1 & $\mathrm{P}$ \\
\hline 145792 & $\mathrm{AB}$ & 6.31 & 8.07 & 0.02 & 0.62 & 0.02 & & 1.693 & 0.018 & 219.8 & 0.2 & 2 & $\mathrm{P}$ \\
\hline 147165 & $\mathrm{AC}$ & 2.61 & 4.77 & 0.07 & 0.06 & 0.07 & + & 0.469 & 0.006 & 244.4 & 0.4 & 1 & $\mathrm{P}$ \\
\hline 151890 & $\mathrm{AP}$ & 4.22 & 10.33 & & 2.02 & & $\mathrm{c}$ & 9.154 & 0.092 & 210.2 & 0.1 & 1 & P? new \\
\hline 157056 & $\mathrm{AB}$ & 3.93 & 5.02 & 0.24 & & & & 0.300 & 0.025 & 251.6 & 3.3 & 1 & $\mathrm{P}$ \\
\hline
\end{tabular}

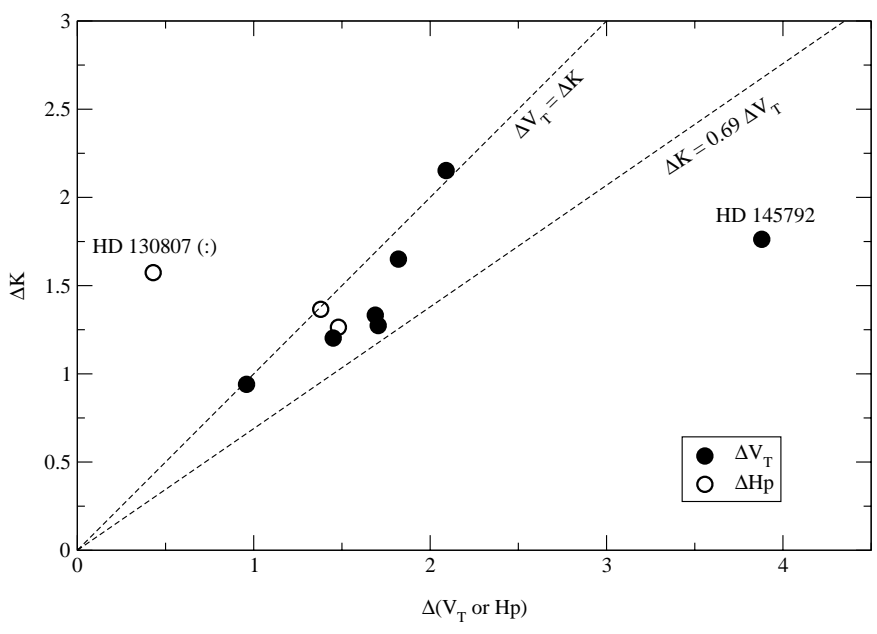

Fig. 5. Comparison of the magnitude differences in the $K$ band with visual magnitude difference from Hipparcos $(\Delta \mathrm{Hp})$ or Tycho $\left(\Delta V_{\mathrm{T}}\right.$, Fabricius \& Makarov 2000). The leftmost point represents the closest $\left(\rho \approx 00^{\prime \prime} 1\right)$, unreliably measured pair HD 130807; the rightmost HD 145792 is possibly an infrared companion. The upper line represents the equal differences whilst the lower the ones expected from the standard relations (Lang 1992).

latter object and HD $100841 \mathrm{P}$ need more observations to clarify their true nature.

\section{Statistics of companions}

\subsection{The color-magnitude diagram}

The color-magnitude diagram is presented in Fig. 6 where only the stars observed under photometric conditions are plotted. The $J$ magnitude is taken as a photospheric luminosity indicator since it is known to be relatively free of IR excess, unlike the $K$ magnitude.

The Main Sequence (MS) is traced from the data of Lang (1992) for a fixed distance modulus of 5.73 $(140 \mathrm{pc})$. The primaries fall mostly near MS or scatter to the right from MS. The highly deviating point belongs to HD 100546 (Sect. 3.3). In general, the measured $J-K$ colors are validated by this plot.

The isochrones for $3 \mathrm{Myr}$ and $10 \mathrm{Myr}$ ages are based on the data of d'Antona \& Mazzitelli (1994, DM94) (for lowmass stars, we used their tracks computed with Alexander, Rodgers and Iglesias opacities and CM convection, shown to correspond to real PMS stars by a number of authors). Triangles mark the masses of $1.5,1.0,0.7,0.5,0.3,0.2$, $0.1,0.08$ solar masses. The effective temperatures $T_{\mathrm{e}}$ and bolometric luminosities were converted to the observed $(J, J-K)$ parameters. This transformation is not precise, involving some assumptions; the tracks themselves are not quite secure, neither. 


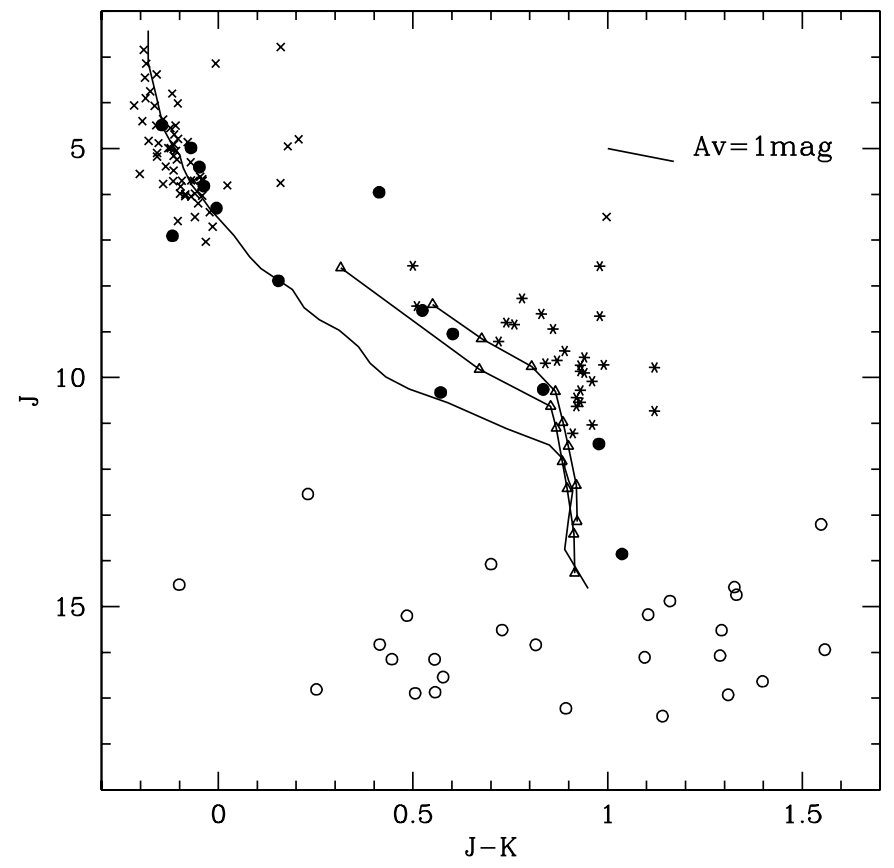

Fig. 6. The color-magnitude diagram. Filled circles - physical secondaries, empty circles - optical secondaries, crosses - primaries. Only the stars with valid photometry are plotted. The long curve is the Main Sequence for a distance modulus of 5.73, extending from B0V to M6V. The $3 \mathrm{Myr}$ and $10 \mathrm{Myr}$ isochrones for masses from 1.5 down to 0.08 solar mass are plotted as well (lines with triangles). Asterisks - low-mass population of the Sco OB2 association from (Walter et al. 1994). A short segment shows the reddening vector for $A_{V}=1^{\mathrm{m}}$.

New tracks were published by a number of authors recently. Among them, the work of Baraffe et al. (1998) is most useful, giving the synthetic absolute $J$ and $K$ magnitudes instead of $T_{\mathrm{e}}$ and bolometric luminosities. Thus, the comparison with observations is more direct. We found that new isochrones do not differ from those of DM94 significantly. Although the slim available data on PMS masses seem to support the Baraffe et al. tracks (Steffen et al. 2001), the new tracks do not reproduce well the mass-luminosity $(M-L)$ relation of older MS stars.

The actual colors and magnitudes of the low-mass PMS population in Sco OB2 are displayed in Fig. 6 as asterisks using the data of Walter et al. (1994). They estimated that the masses of these X-ray selected stars range from 0.2 to 2 solar, the typical extinction is $A_{V} \approx 0.5$, and the age is around $1 \mathrm{Myr}$. We express some reservations about this age estimate, since OB stars are much older (de Zeeuw et al. 1999).

\subsection{Status of the secondary components}

The secondaries known to be physical are either on MS or above. Some newly discovered components also fall within the low-mass zone and can be classified as physical. On the other hand, most of the faint secondaries are optical. The lower limit for physical secondaries corresponds roughly to $J=13$ or $K=12$ (for $3 \mathrm{Myr}$ age). Some secondaries have $J-K>1.7$, falling outside the graph boundary. We presume that they are heavily reddened background stars.

All previously known components are considered here as physical. The reason for this is that the Sco OB2 association has a relatively large proper motion of 40 mas/yr, and any background component would show up by its fast motion relative to primary. This argument does not apply, however, to the association members that project close to the targets.

All new components which are fainter than $K=12$ or $J=13$ are considered as optical. The remaining bright opticals are identified on the color-magnitude diagram when valid photometry is available. Otherwise, an uncertain status is assigned. On the total, there are 37 physical components, of which 10 are new ( 3 of them have uncertain status and 1 is the questionable star HD $100841 \mathrm{P}$ ). The total number of new optical components is 70 .

Physical and optical components show quite distinct distributions of angular separations, with maxima at small and large separations, respectively. We take this as an indirect evidence that our photometric classification is statistically correct.

The status codes in Table 2 are:

$\mathrm{P}$ - for physical companions;

P? - for uncertain physical companions;

$\mathrm{O}$ - for definitely optical (background) companions;

O? - for likely optical companions.

The part of Table 2 reproduced in this paper gives information on all observed non-optical companions. The discrimination between the optical and physical secondaries is one of the important issues in this study and potentially a weak point. Naturally, all new components have small masses and their classification directly affects the lower bin of the mass ratio distribution. In Sect. 4.5 we consider the 3 uncertain companions as physical. Our guess is that 1 or 2 of them may be optical, but this revision would only reinforce our main conclusions.

\subsection{Statistics of background sources}

In Fig. 4 the cumulative distribution of optical components (number of components $N$ that are brighter than a given magnitude) is plotted in solid line. Only components with $\rho>3^{\prime \prime}$ were selected (at smaller separations, the companion detection in the coronographic frames is affected by the halo of the primary). The dashed line shows the same distribution for the sky fields. Only companions detected in both $J$ and $K$ bands were selected.

The two curves coincide to within statistical fluctuations, especially in the important region around $K=$ 12-14 where the discrimination between optical and physical components is critical for our analysis. We note that the total surface of the main fields is smaller than the surface of the sky fields by $15 \%$ (central $3^{\prime \prime}$ excluded). On the other hand, the detection limits in the sky fields may be lower because of the lower Strehl ratio (anisoplanatism). All in all, there are 5 components brighter than $K=12$ in 
the sky fields and 3 such optical components in the main fields. Taken at face value, it means that about 2 components in the main fields may be still misclassified as physical. However, it is clear that our classification scheme did not miss a large number of faint physical companions, otherwise we would observe an excess of "opticals" in comparison with the sky fields.

An important feature of the background sources is their highly fluctuating density. For 45 targets, optical components were found neither in the main nor in the sky fields; on the other hand, in the remaining fields a significant correlation between the number of optical components in the main and sky fields was found. In 3 main fields, as much as 7 to 9 optical components were detected, with no less than 6 components in the corresponding sky fields. This correlation is a strong argument for the optical nature of faint components.

Quite often, more than one component identified as optical are present in the main fields and have comparable separations. They can not be physical for yet another reason: non-hierarchical stellar systems are dynamically unstable and must disintegrate, given the age of Sco OB2 group.

In the following sub-sections we consider the calculation of the mass ratio $q=M_{2} / M_{1}$. It will allow us to derive the mass ratio distribution $f(q)$ of physical systems.

\subsection{Estimation of masses and mass ratios}

In principle, the masses of MS stars can be estimated from their $J-K$ colors. However, the colors are measured with large errors and are distorted by extinction and IR excess. Hence, the best way to estimate the masses of the companions is to use their luminosities, preferably in the $J$ band. On the other hand, the use of the mass-luminosity (M-L) relation requires a knowledge of distance and age (for low-mass stars) and it relies also on the yet uncertain PMS tracks.

The $M-L$ relations for MS and PMS stars are taken from Lang (1992) and Baraffe et al. (1998), respectively. For PMS tracks, the mass $M$ depends on $A$ (the logarithm of age in Myr) and absolute $J_{0}$ or $K_{0}$ magnitudes approximately as

$$
\begin{aligned}
& \lg \left(M / M_{\odot}\right) \approx 0.706-0.305 J_{0}+0.545 A \\
& \lg \left(M / M_{\odot}\right) \approx 0.440-0.313 K_{0}+0.581 A .
\end{aligned}
$$

For MS stars, the $M-L$ relation was approximated by several linear segments. The accuracy of all these approximations is better than $\pm 10 \%$ in mass. Of course, the actual isochrones are not linear but rather "saturate" as the luminosity reaches its MS value. So, the companion masses were calculated from both MS and PMS relations and the lowest of the two values was taken. The actual ages of subgroups were used in the calculations: 4.5 Myr for Upper Scorpius, 14.5 Myr for Upper Centaurus-Lupus

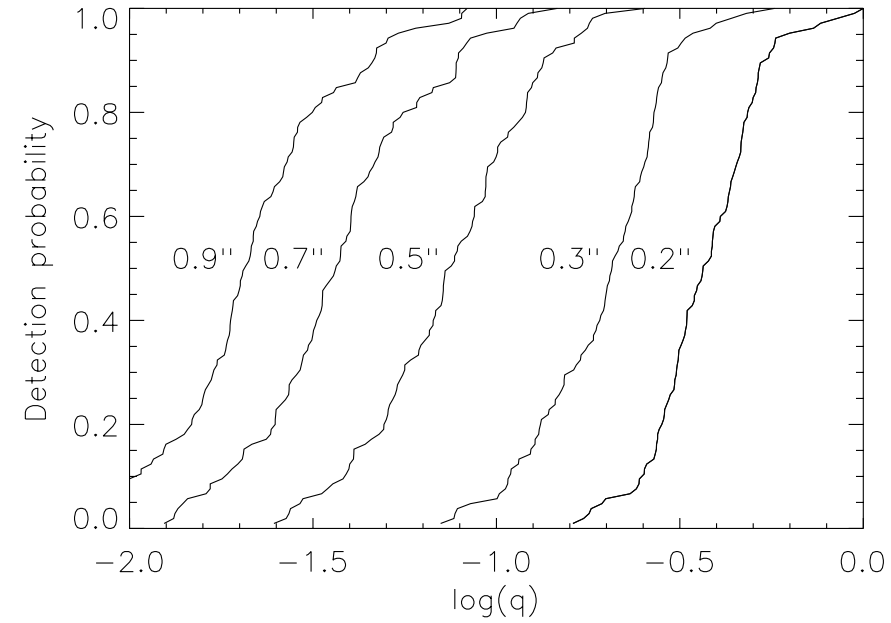

Fig. 7. The fraction of detectable components as a function of mass ratio $q$ for separations from $0 . \prime 2$ to 0. .' 9 , as indicated by the numbers near the curves.

and 11.5 Myr for Lower Centaurus-Crux (de Zeeuw et al. 1999).

The $M-L$ relation is favorable for the mass estimation: its slope at MS roughly corresponds to $M \propto L^{0.5}$ and steepens to $M \propto L^{0.8}$ for the PMS tracks. This explains why the mass estimates are relatively insensitive to data reduction details.

The masses of the primary stars were also estimated from their luminosities rather than from their spectral classes. This was done to cancel as much as possible the influences of errors in distances, extinction, etc., which affect the masses of primaries and secondaries in almost the same way and hence have little effect on the mass ratio $q$. Even the errors in the photometry caused by nonphotometric conditions are compensated to some extent because $q$ depends mostly on the magnitude difference.

The detection limits were studied and modeled in Sect. 3.2. We converted the derived log-linear relations between $K_{\lim }$ and $R=\lg \left(\rho / 1^{\prime \prime}\right)$ into the limiting mass ratio $q_{\text {lim }}$ using the $M-L-$ Age relation in the $K$ band and the actual age of each target. The $K$-band is used since lowmass red companions are better detected at longer wavelengths. These limiting mass ratios are sorted in increasing order and plotted in Fig. 7 as detection probability (bias). If all frames were taken in exactly the same conditions, all $q_{\text {lim }}$ would be identical. The actual distribution of $q_{\text {lim }}$ reflects the spread in the observing conditions, exposure time, target brightness, etc.

The detection bias is modeled as a set of linear functions of $\lg q$, neglecting the "tails" of the distributions in Fig. 7. Linear models are defined by two parameters, the $\lg q_{\text {lim }}$ where $50 \%$ of companions are detected and the full range in $\lg q_{\mathrm{lim}}$. These parameters were represented by quadratic functions of $R$. The analytical model of detection bias is plotted in Fig. 8. 


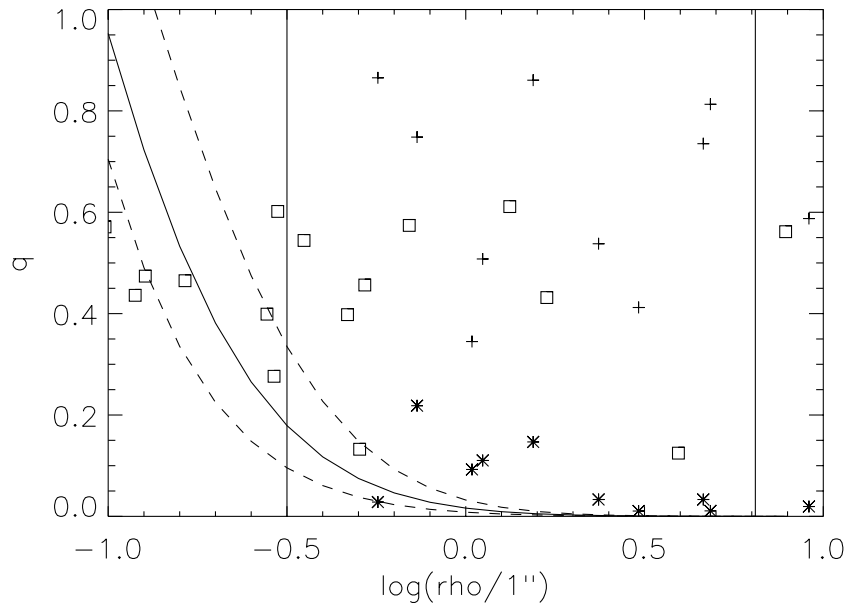

Fig. 8. The distribution of the known and measured (squares), known and unmeasured (pluses) and newly discovered (asterisks) physical components in the $(\lg \rho, q)$ plane. The detection model is shown by the solid line ( $50 \%$ detection) and dashed lines ( $0 \%$ and $100 \%$ detection). The separation range selected for statistical analysis is delimited by the vertical lines.

\subsection{Mass ratio distribution and companion fraction}

The distribution of the physical secondaries in the $(\lg \rho, q)$ plane is shown in Fig. 8. It is expected that the component distribution in $\lg \rho$ should be uniform (Öpik's law). Indeed, it seems to be the case. Moreover, there seems to be no significant correlation between $\rho$ and $q$ in the separation range studied. This permits to discuss the $q$ distribution for all relevant separations jointly.

We limit the statistical analysis to the separation range from 0 "' 3 to 6 ". 4 , which corresponds to $45-900 \mathrm{AU}$ at the distance of Sco OB2. For lower separations, the detection bias in $q$ becomes too important. The upper limit is determined by the half-size of the frames. Components at larger separations were actually detected in the corners, but, as it is evident from Fig. 8, little can be gained by extending the limit to $9^{\prime \prime}$ and making corrections for incomplete surface coverage.

A total of 27 physical components fall in the selected separation range which covers 1.3 decades. Assuming that the distribution in $\lg \rho$ is uniform and that the distribution in $q$ is smooth, we estimate the fraction of missed components by integrating the bias model within the selected limits for each bin of the $q$ histogram. The fraction of detected components is more than 0.8 for all bins, which means that our incompleteness correction remains reasonably small. The resulting histogram of $q$ (after correction for incompleteness) is plotted in Fig. 9 (left).

The same data reduction steps were done for the DM94 tracks and two fixed ages of 3 and $10 \mathrm{Myr}$. The results are qualitatively very similar. Comparing the histograms for these two isochrones, we saw that assuming a younger age results in lower masses, slightly re-distributing the components between the two lowest bins.

In Fig. 9 (right) the same histogram is plotted as cumulative distribution, in order to avoid binning. It is corrected for detection incompleteness by increasing the "weights" of low- $q$ systems accordingly. The slope of the cumulative distribution clearly increases towards low $q$. Adopting the power law $f(q) \propto q^{-\Gamma}$, it seems that the index $\Gamma=0.5$ fits the data well.

The $q$-distribution does grow towards low $q$, but only mildly. On the other hand, the power law with index $\Gamma$ from 1.8 to 2.1 is clearly rejected. Such power law corresponds to the initial mass function (IMF) in Sco OB2 (Brown 1998; Preibisch \& Zinnecker 1999; Preibisch et al. 2001) and would apply if the secondary components were selected randomly from IMF.

The power-law distributions $f(q) \propto q^{-\Gamma}$ are not integrable for $\Gamma>1$, in this case the total binarity is determined by the elusive cut-off at low $q$. On the contrary, the actual distribution is smooth and integrable, the total binarity is well defined. The total number of components (after correction for incompleteness) is 29.6 for our 115 targets and in the separation range of 1.3 decades. This leads to a companion star fraction $(C S F)$ of $0.20 \pm 0.04$ per unity of the logarithm of separation (decade of $\rho$ ) or $0.13 \pm 0.02$ per decade of period.

We repeated the analysis while excluding the 3 faint components with uncertain physical status and the 2 components with insecure detection. The number of companions in the $0^{\prime \prime} 3-6^{\prime \prime}$ separation range becomes 23 (24.85 after correction for incompleteness), the CSF is revised down to $0.17 \pm 0.04$. The lowest bin in the histogram (Fig. 9 left) becomes $30 \%$ less, leading to even more uniform $f(q)$ which may be approximated by a $q^{-0.3}$ law. This exercise shows that our conclusions do not critically depend on the remaining uncertainties in the experimental data.

\section{Discussion}

Before our study, we expected the number of unknown low-mass visual components around B-type stars to be large, because low-mass stars are, generally, much more frequent than high-mass stars, and because the detection of such components by traditional techniques was difficult. Now we see that the newly detected low-mass physical components are not so numerous and that the old detections were essentially complete down to at least $q=0.3$. It was indeed necessary to go much deeper in magnitude difference to validate the historical data. In this perspective, the fact that most of our newly detected components are optical is not disappointing.

Our result is in marked disagreement with the conclusions of Abt et al. (1990) who claim that the distributions of the secondary components of B2-B5 stars follows the Salpeter mass function and increases steeply towards small $q$ in the range of separations studied here. Their analysis is based on the known visual components confirmed by common proper motions. Still, we strongly suspect that most of the wide pairs in the B2-B5 sample of Abt et al. are optical. In their Table 5 there are 7 trapezium-type systems with separations in the $10^{\prime \prime}$ to $63^{\prime \prime}$ range and separation 

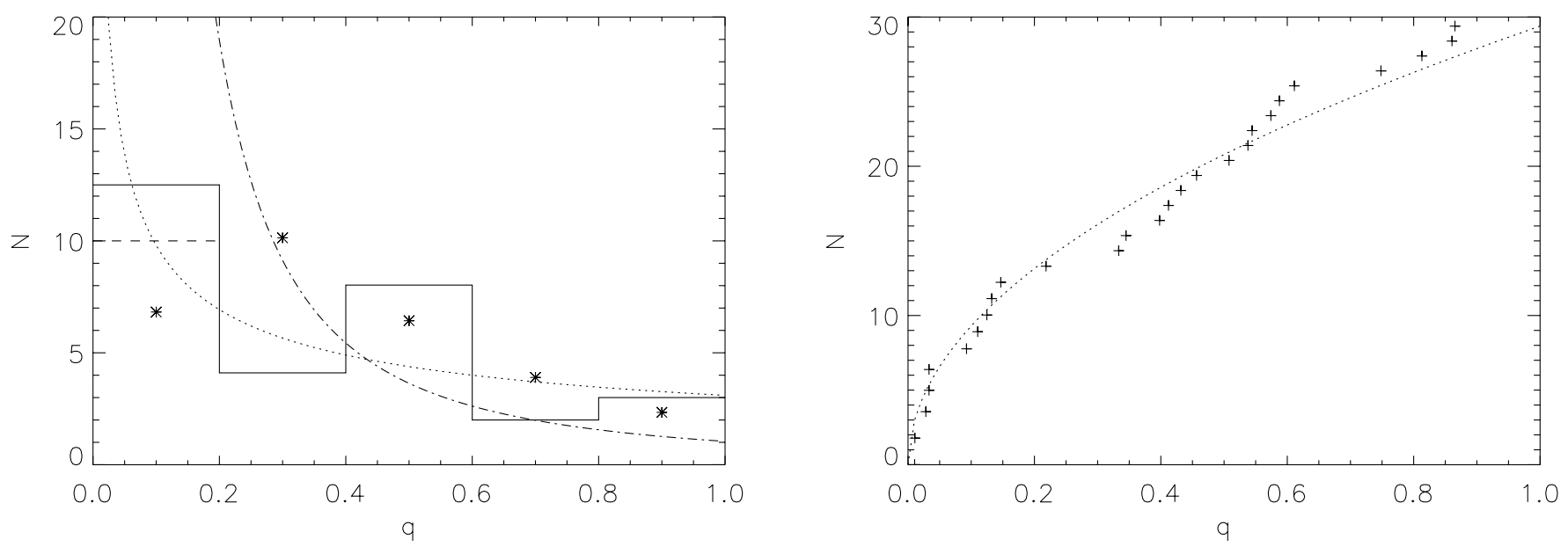

Fig. 9. Left: the histogram of the mass ratio distribution $f(q)$ in separation range from 45 to 900 AU (full line). The dashed line shows the uncorrected value in the first bin. Asterisks - re-normalized $f(q)$ of G-type dwarfs with periods $P>30$ yr $(a>10 \mathrm{AU})$ from DM91. Dotted line corresponds to $f(q) \propto q^{-0.5}$, dash-dot - to $f(q) \propto q^{-1.8}$. Right: cumulative distribution corrected for detection incompleteness (crosses) and its modeling as $F(q)=q^{0.5}$.

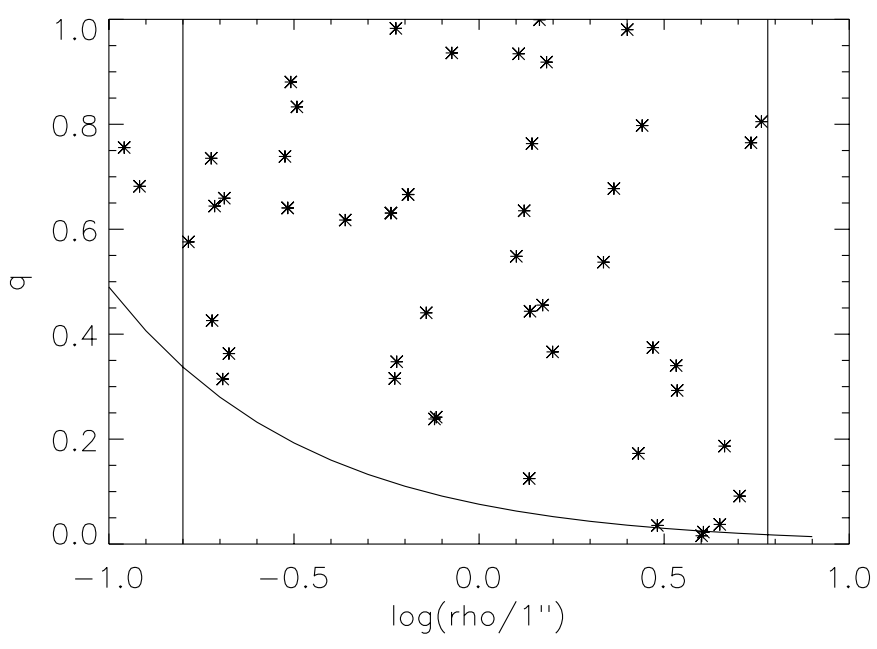

Fig. 10. Mass ratio as a function of separation for the 56 lowmass PMS binaries in Sco OB2 studied by Köhler et al. (2000). The curve shows indicative detection bias, vertical lines indicate the separation range of their statistical analysis.

ratio less than 3 which are likely unstable, if physical. When the spectra of the components of 116 trapeziumtype systems were taken by Abt \& Corbally (2000), they discovered that only 28 of them can be physical - a proof that most of the cataloged trapezia are indeed spurious.

In Table 4 we give a summary of the statistical binarity studies in different populations, for comparison with our results. The sample size $N$ and the approximate range of separations surveyed are indicated. The CSF is only a weak function of separation, hence it is legitimate to compare results in different separation ranges. For the Hyades, the CSF given by Patience et al. (1998) involved a factor of 2 correction for undetected systems, hence we preferred the uncorrected lower limit.

The most recent study of the multiplicity of low-mass population of Sco OB2 (Köhler et al. 2000) is very similar to the present work by the number of targets surveyed
Table 4. Companion star fraction (number of companions per primary per decade of separation) in different populations.

\begin{tabular}{|lccrc|}
\hline $\begin{array}{l}\text { Spectral type, } \\
\text { environment }\end{array}$ & $N$ & $\begin{array}{c}\text { Range, } \\
\text { AU }\end{array}$ & CSF & Ref. \\
\hline B, Sco OB2 & 115 & $45-900$ & $0.20 \pm 0.04$ & 1 \\
PMS, Sco OB2 & 118 & $20-900$ & $0.21 \pm 0.04$ & 2 \\
PMS, Sco-Lup & 269 & $120-1800$ & $0.12 \pm 0.02$ & 3 \\
PMS, Tau-Aur & 104 & $120-1800$ & $0.22 \pm 0.04$ & 3 \\
G, field & 164 & $40-900$ & $0.12 \pm 0.03$ & 4 \\
M, field & 58 & $10-1000$ & $0.11 \pm 0.04$ & 5 \\
A-K, Hyades & 167 & $5-50$ & $>0.16 \pm 0.03$ & 6 \\
G-K, Pleiades & 144 & $12-1000$ & $0.14 \pm 0.02$ & 7 \\
G-K, Praesepe & 149 & $15-600$ & $0.15 \pm 0.03$ & 8 \\
\hline
\end{tabular}

References: 1 - this work; 2 - Köhler et al. (2000); 3 - Brandner et al. (1996); 4 - Duquennoy \& Mayor (1991); 5 - Fisher \& Marcy (1992); 6 - Patience et al. (1998); 7 - Bouvier et al. (1997); 8 - Bouvier et al. (2001).

and the separation range $\left(0^{\prime \prime} 13-6^{\prime \prime}\right)$. They find a $C S F$ of $0.21 \pm 0.04$ per decade of separation, indistinguishable from our result. We tried to process the magnitudes and flux ratios of the 56 systems from their Tables 2 and 3 in the same manner as our data, converting the $K$ magnitudes into mass ratios with the help of a $3 \mathrm{Myr}$ isochrone and for the assumed distance of $140 \mathrm{pc}$. The results are shown in Fig. 10. The curve indicates our best guess of the detection threshold, which is $\sim 3$ times higher than the threshold given by the authors themselves. About 7.8 systems in their sample (mostly with large separations) were estimated to be optical. Taking into account these uncertainties, it does not make sense to compare the histograms of $q$. All that can be said is that the $q$ distribution seems to be uniform and certainly does not increase towards small $q$ as much as it would be expected from the IMF slope.

Brandner et al. (1996) provided a comprehensive summary of the previous binarity studies among the PMS stars which were made in the visible. The $C S F$ in the 
Upper Scorpius and Lupus is $0.12 \pm 0.02$ per decade of separation for a combined sample of 269 stars. The global CSF among 525 PMS stars is $0.14 \pm 0.013$, which remains the most statistically sound estimate to date (however, in the Taurus-Auriga region the $C S F$ is $0.22 \pm 0.04)$. Clearly, Köhler et al. obtained a significantly higher $C S F$ for the same population. However, Brandner et al. find an evidence for CSF variations across the Sco OB2, and the regions studied by Köhler et al. happen to be near the binary-rich zone. This seems to be the most plausible explanation of this discrepancy.

Our sample covers a large region in the sky. For this reason the $C S F=0.12$ measured by Brandner et al. is more appropriate for comparison with our result, $C S F=0.20$. Thus, more massive B-type stars do have an increased $C S F$ with respect to the lower-mass PMS stars. The same conclusion is reached by comparing our result to the binary fraction of low-mass field dwarfs and low-mass cluster population (Table 4 ).

The unbiased mass ratio distribution for visual binaries with B-type primaries is the main result of our study. The idea of independent selection of the visual components from some initial mass function can now be definitely rejected. However, the new result is not so unexpected, after all. The $f(q)$ obtained by DM91 for the wide $(\lg P$ (days) $>4)$ systems is similar to the $f(q)$ found here (Fig. 9). The mass ratio should be indeed biased towards a uniform one by stellar dynamics, whatever the IMF. N-body simulations demonstrated that the shape of $f(q)$ depends on the density and composition of the stellar aggregate where the binaries have been formed, and a certain choice of parameters may reproduce the result of DM91 (Kroupa 1995; Durisen et al. 2001).

On the other hand, the secondary components of B-type stars must have been formed in the same clouds as their primaries, in conditions that likely favored high masses. The secondaries may thus be distinct from the rest of low-mass population in Sco OB2 with respect to their initial mass function and age. For the moment we are not able to disentangle the influence of dynamical and birth factors on the final mass ratio distribution. The important thing is that the distribution itself is now known with some confidence.

\section{Appendix A: Detection and measurement of close companions with DAOPHOT}

In this appendix we present the solution to the problem of PSF selection for DAOPHOT fitting in non-coronographic mode. A simple subtraction of the radial profile of the PSF (jupe algorithm) leaves the bright semi-static speckle pattern around the target star unattenuated. To remove it partially, we produced the set of average PSFs for each night and each filter, selected by the value of the image Strehl ratio into 3-5 groups. Being averaged over images of many objects, these synthetic PSFs contain no trace of any possible faint companions which hide under the speckle pattern (the extra PSF cleaning function of DAOPHOT
II removes all outlying features in the same way as median filtering). The radius of these synthetic PSFs is 35 pixels = 1.'75 (maximal available in DAOPHOT from the release of ESO-MIDAS which we used).

Each non-coronographic image of the target star was fitted with one of the average PSFs with similar Strehl ratio. The residuals after the PSF subtraction were visually searched for new close companions. The companions of both newly found and known close $(\rho<1$ 1.75) visual systems were simultaneously fitted using NSTAR or ALLSTAR utility of DAOPHOT to produce the differential astrometric and photometric measurements reported in this paper.

To get an estimate of the detection limits achieved with the subtraction of the synthetic PSFs, we also used the jupe program. After subtraction, the intensity of the remaining speckle noise decreased to a level which coincided with the detection limit in the coronographic images (Fig. 3).

\section{Appendix B: Differential astrometry with coronograph}

The coordinate differences between the source and target stars were measured in three different ways. The first and most reliable method is the simultaneous PSF fitting to primary and secondary stars in the non-coronographic images.

However, the simultaneous PSF fitting is not applicable to coronographic images since the primary star is not visible. Instead, the position of the primary was determined from the PSF wings by the jupe program. The relation between this method and direct PSF fitting was studied. The precision of the jupe coordinates of primaries was found to be \pm 0.3 pixels, with a constant bias of -1.5 and -0.6 pixels in the $x$ and $y$ directions, respectively. This bias is caused, possibly, by the asymmetry of the PSF wings.

Alternatively, the known positions of the primary in the two quadrants of the non-coronographic images can be used to predict the position of the star under the mask, supposing that AO system stabilizes the image in the detector plane and that the offsets provided by the chopping mirror of ADONIS are precise and repeatable. These offsets were studied and calibrated. It turned out that the chopping mechanism moves the target star across the detector with a rms error of about \pm 0.25 pixels; the error never exceeds 0.6 pixels.

Whenever components were measured by several methods, the resulting coordinates were computed as weighted averages, with a weight equal to 10 for direct PSF fitting, weight 1.5 for jupe coordinates and weight 2.0 for coordinates extrapolated from non-coronographic images.

Acknowledgements. The Fellowship of the Belgian Services Fédéraux des Affaires Scientifiques, Techniques and Culturelles provided the possibility for N.S. to work at the 
Royal Observatory of Belgium, thanks to the support of E. van Dessel. Authors are grateful to the staff of the ESO $3.6 \mathrm{~m}$ telescope, especially to O. Marco, for their support of observations. Thanks to A. Chalabaev for his help with the data acquisition and stimulating discussions. Th. Nakos and A. Whiting have kindly conceded to correct our English. This research made use of the Simbad database operated at CDS, Strasbourg, France and of the Digital Sky Survey produced at the Space Telescope Science Institute, USA.

\section{References}

Abt, H. A., Gomez, A., \& Levy, S. C. 1990, ApJS, 74, 551

Abt, H. A., \& Corbally, C. J. 2000, ApJ, 541, 841

Augereau, J. C., Lagrange, A. M., Mouillet, D., et al. 2001, A\&A, 365, 78

Baraffe, I., Charbier, G., Allard, F., \& Hauschild, P. H. 1998, A\&A, 337, 403

Beuzit, J.-L., Mouillet, D., Lagrange, A.-M., et al. 1997, A\&AS, 125,175

Bouvier, J., Rigaut, F., \& Nadeau, D. 1997, A\&A, 323, 139

Bouvier, J., Duchêne, G., Mermilliod, J.-C., \& Simon, T. 2001, A\&A, 375, 989

Brandner, W., Alcalá, J. M., Kunkel, M., et al. 1996, A\&A, 307,121

Brown, A. G. A. 1998, in The Stellar Initial Mass function, ed. G. Gilmore, \& D. Howell, ASP Conf. Ser., 142, 45

Brown, A. G. D., \& Verschueren, W. 1997, A\&A, 319, 811

D'Antona, F., \& Mazzitelli, I. 1994, ApJS, 90, 467

Devillard, N. 1997, The Eclipse software, The Messenger, No. 87

de Geus, E. J., de Zeeuw, P. T., \& Lub, J. 1989, A\&A, 216, 44

Duchêne, G. 1999, A\&A, 341, 547

Duquennoy, A., \& Mayor, M. 1991, A\&A, 248, 485 (DM91)

Durisen, R. H., Sterzik, M. F., \& Pickett, B. K. 2001, A\&A, 371,952

ESA 1997, European Space Agency, SP-1200
Fabricius, C., \& Makarov, V. V. 2000, A\&A, 356, 141

Fischer, D. A., \& Marcy, G. W. 1992, ApJ, 396, 178

Hubrig, S., Le Mignat, D., \& Krautter, J. 2001, A\&A, 372, 152

Köhler, R., Kunkel, M., Leinert, C., \& Zinnecker, H. 2000, A\&A, 356, 541

Kroupa, P. 1995, MNRAS, 277, 1491

Lang, K. R. 1992, Astrophysical data, Planets and Stars (Berlin: Springer-Verlag)

Mason, B. D., Gies, D. R., Hartkopf, W. I., et al. 1998, AJ, 115,821

Meeus, G., Waters, L. B. F. M., Bouwman, J., et al. 2001, A\&A, 365, 476

Otero, S., Fraser, B., \& Lloyd, Ch. 2001, IAU Inform. Bull. Var. Stars, 5026, 1

Patience, J., Ghez, A. M., Reid, I. N., et al. 1998, AJ, 115, 1972

Preibisch, T., \& Zinnecker, H. 1999, AJ, 117, 2381

Preibisch, T., Guenther, E., \& Zinnecker, H. 2001, AJ, 121, 1040

Racine, R., Walker, G. A. H., Nadeau, D., et al. 1999, PASP, 111,587

Söderhjelm, S. 1997, in Visual Binary Stars: Formation, Dynamics and Evolutionary Tracks, ed. J. A. Docobo, A. Elipe, \& H. McAlister (Kluwer), 497

Söderhjelm, S. 2001, Astr. Nachr., 321, 165

Steffen, A. T., Mathieu, R. D., Lattanzi, M. G., et al. 2001, AJ, 122, 997

Stetson, P. 1987, PASP, 99, 191

Tokovinin, A. A., Chalabaev, A., Shatsky, N. A., \& Beuzit, J. L. 1999, A\&A, 346, 481 (TCSB99)

Walter, F., Vrba, F. J., Mathieu, R. D., et al. 1994, AJ, 107, 692

Weigelt, G., Balega, Yu., Preibisch, Th., et al. 1999, A\&A, 347, L15

de Zeeuw, P. T., Hoogerwerf, R., Bruijne, J. H. J., et al. 1999, AJ, 117, 354 